\title{
A Simple Electrocardiographic Predictor of Early Recovery of Left Ventricular Function in Stress Cardiomyopathy
}

\author{
Kshamaya B. Panchamukhi*, Orlando Santana, Cesar A. Conde, Gervasio A. Lamas
}

Mount Sinai Medical Center, Department of Cardiology, Columbia University, Miami Beach, USA.

Email: *kshamaya@yahoo.com

Received September $18^{\text {th }}, 2012$; revised October $20^{\text {th }}, 2012$; accepted November $30^{\text {th }}, 2012$

\begin{abstract}
Objective: To identify electrocardiographic parameters that predict extent of early improvement in ejection fraction (EF) in patients with stress cardiomyopathy. Methods: We collected baseline clinical, ECG and imaging data and follow up echocardiography data on 30 consecutive patients with stress cardiomyopathy. The relationship between baseline ECG parameters and Delta EF was evaluated by univariate and multivariable analysis. Results: Median EF improved from $35 \%$ to $55 \%(\mathrm{p}<0.0001)$. Delta EF showed negative correlation with number of leads with T inversion $(\mathrm{p}$ $=0.01)$ and QT interval $(\mathrm{p}=0.02)$. The number of leads with $\mathrm{T}$ inversion was independently associated with Delta EF (coefficient $-4.878, \mathrm{p}=0.04$ ). Conclusion: The number of leads with $\mathrm{T}$ wave inversion is negatively correlated with and is an independent predictor of the extent of early improvement of EF in patients with stress cardiomyopathy. Hence the ECG at initial presentation can be used as a simple tool to predict LV function recovery.
\end{abstract}

Keywords: Ejection Fraction; Cardiomyopathy; Electrocardiography

\section{Introduction}

Stress cardiomyopathy is a reversible cause of left ventricular (LV) dysfunction [1]. However, baseline predictors of LV function recovery have not been specifically addressed by previous studies. The purpose of this study was to identify baseline parameters that predict extent of improvement in ejection fraction (EF) early after presentation.

\section{Method}

We conducted a combined retrospective and prospective study of 30 consecutive patients who met Mayo Clinic criteria for stress cardiomyopathy [1] from 2005 to 2008 at our institution. Cases were carefully reviewed and selected based on a consensus between two of the authors (KBP, OS).

Inclusion criteria were: 1) transient hypokinesis, akinesis or dyskinesis of the left ventricular mid segments with or without apical involvement and regional wallmotion abnormalities not conforming to a single epicardial vascular distribution 2) new electrocardiographic abnormalities including ST-segment elevation, ST segment depression or T-wave inversion or elevation of car-

*Corresponding author. diac biomarkers.

Exclusion criteria were: 1) significant obstructive coronary disease or angiographic evidence of acute plaque rupture; 2) myocarditis; 3) pheochromocytoma; 4) intracranial process.

Baseline data collection included clinical history, physical examination, laboratory and ECG findings, echocardiography and cardiac catheterization data. Patients were followed up with repeat echocardiography. The study was approved by the Institutional Review Board.

ECG data: pertinent ECG data collected included: presence of ST depression, number of leads with ST depression, and maximum amplitude of ST depression in millimeters (mm). Similar data was recorded for ST elevation and $\mathrm{T}$ wave inversion. The diagnostic criteria for ST depression were: J-point depression of $0.5 \mathrm{~mm}$ in leads $V_{2}$ and $V_{3}$ and $1 \mathrm{~mm}$ in all other leads. The diagnostic criteria for ST elevation were as follows - for men, J-point elevation in 2 contiguous leads measuring $2 \mathrm{~mm}$ in leads $V_{2}$ and $V_{3}$ and $1 \mathrm{~mm}$ in all other leads and for women, J-point elevation in 2 contiguous leads of 1.5 $\mathrm{mm}$ in leads $\mathrm{V}_{2}$ and $\mathrm{V}_{3}$ and $1 \mathrm{~mm}$ in all other leads [2]. The number of leads with $\mathrm{T}$ wave inversion were counted regardless of ST changes in those leads. QT and corrected QT interval (by Bazett's formula [3] were re- 
corded. Cut-off values for QTc prolongation were 450 milliseconds for men and 460 milliseconds for women [4].

Biomarker data: Peak values of cTroponin I, CK-MB, NTpro-BNP are recorded. The upper reference limit for cTroponin I in our laboratory is $0.07 \mathrm{ng} / \mathrm{ml}$.

Echocardiographic parameters studied on the initial and follow-up examinations included chamber size, left ventricular wall thickness, global ejection fraction, segmental wall motion abnormality, estimated left atrial and pulmonary artery systolic pressure and valve morphology and function. A single observer, blinded to clinical data, read all echocardiograms. Delta EF was defined as follow-up EF minus initial EF. Measurements were performed according to the American Society of Echocardiography Guidelines $[5,6]$.

Cardiac catheterization findings studied were coronary anatomy, left ventricular end-diastolic pressure, left ventricular size, global ejection fraction, segmental wall motion abnormality and mitral regurgitation.

Statistical analysis: Data were expressed as percentages for categorical variables, mean \pm SD for normally distributed continuous variables, and median (interquartile range-IQR), for non-normally distributed continuous variables. Initial and follow up EF were compared using the paired t-test. The relationship between baseline parameters and Delta EF was analyzed using ANOVA and Spearman's correlation. Multivariable linear regression was used to identify independent predictors of Delta EF.

\section{Results}

Demographics and clinical presentation: Patients had an average age of 73.2 (SD 15.1) years and $67 \%$ were women. Hypertension was present in $53 \%$, hyperlipidemia in $63 \%$, diabetes in $27 \%$ and smoking in $30 \%$. Presenting clinical parameters were chest pain in $67 \%$, dyspnea in $20 \%$, palpitation in $37 \%$ and syncope in $7 \%$. A precipitating factor was identified in $84 \%$ of patients (Table 1).

Electrocardiographic data: The mean heart rate was 95 (SD 24). ST depression was noted in $43 \%$, ST elevation in $30 \%$, T wave inversion in $70 \%$ and QT prolongation in $60 \%$. Data on number of leads with ST depression, ST elevation and T wave inversion is presented in Table 2 and Figure 1.

Biomarkers: Troponin I was elevated in $60 \%$ of patients, CK-MB in $40 \%$ and NTpro-BNP in $37 \%$. At least one biomarker was elevated in $67 \%$ of patients. The median (IQR) peak values for troponin I, CPK, CK-MB and NT pro-BNP are presented in Table 2.

Cardiac catheterization (Figure 2): There was no significant obstructive lesion on coronary angiogram. The
Table 1. Demographics and clinical presentation.

\begin{tabular}{cc}
\hline Age, mean (SD) years & $73.2(15.1)$ \\
\hline Females, \% & 69 \\
Hypertension, \% & 55 \\
Hyperlipidemia, \% & 65 \\
Diabetes, \% & 7 \\
Smoking, \% & 31 \\
Chest pain, \% & 69 \\
Dyspnea, \% & 40 \\
Palpitation, \% & 38 \\
Syncope, \% & 7 \\
Heart failure, \% & 38 \\
\hline
\end{tabular}

Table 2. Electrocardiographic and biomarker data.

\begin{tabular}{|c|c|}
\hline Heart rate, mean (SD) bpm & $95(24)$ \\
\hline ST depression, $\%$ & 45 \\
\hline ST elevation, $\%$ & 31 \\
\hline $\mathrm{T}$ wave inversion, $\%$ & 73 \\
\hline QT prolongation, $\%$ & 62 \\
\hline $\begin{array}{l}\text { Number of leads with ST depression, } \\
\text { median (IQR) }\end{array}$ & $3(3,6)$ \\
\hline $\begin{array}{c}\text { Number of leads with ST elevation, } \\
\text { median (IQR) }\end{array}$ & $4(3,6)$ \\
\hline $\begin{array}{c}\text { Number of leads with T wave inversion, } \\
\text { median (IQR) }\end{array}$ & $5(4,6)$ \\
\hline QT interval, mean (SD) milliseconds & 404 (SD 80) \\
\hline $\begin{array}{l}\text { Corrected QT interval, mean (SD) } \\
\text { milliseconds }\end{array}$ & 487 (SD 72) \\
\hline Troponin I, median (IQR) & $2.4(\mathrm{IQR} 0.4,5.8)$ \\
\hline CPK, median (IQR) & 173 (IQR 119, 330) \\
\hline CK-MB, median (IQR) & 7.7 (IQR 3.1, 11.7) \\
\hline NTpro-BNP, median (IQR) & 8463 (IQR1, 578, 14, 501) \\
\hline
\end{tabular}

mean left ventricular end-diastolic pressure was 14 (SD 10) $\mathrm{mmHg}$. The median EF by left ventricular angiography was $40 \%$ (IQR 25,45 ).

Initial echocardiography (Table 3, Figure 3): The median ejection fraction was $35 \%$ (IQR 25, 40). Left ventricular hypertrophy was noted in $50 \%$. Diastolic dysfunction was present in $70 \%$ - Grade 1 in $60 \%$ and Grade 2 in 10\%. Estimated left atrial pressure was 14 (SD 5) $\mathrm{mmHg}$ and estimated pulmonary artery systolic pressure was 38 (SD 10). Mitral regurgitation was present 


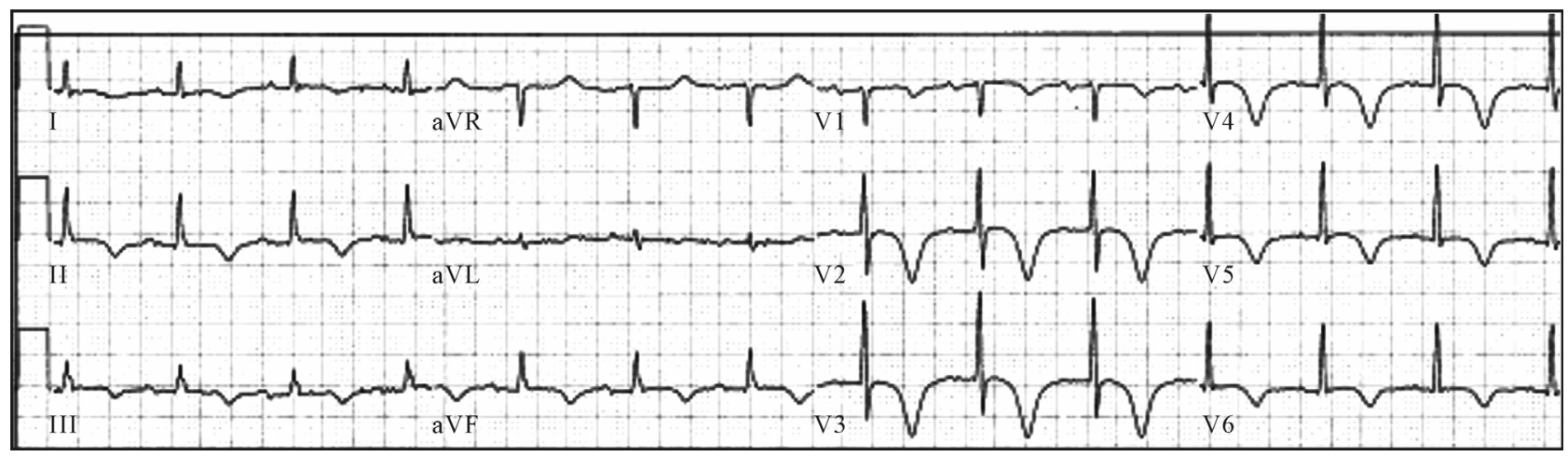

Figure 1. Initial electrocardiogram.

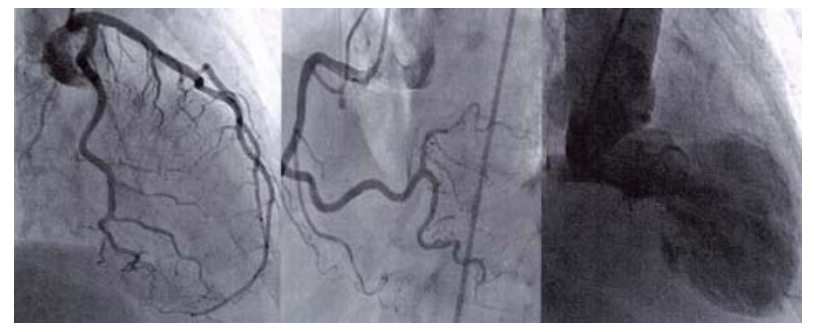

Figure 2. Coronary and left ventricular angiograms showing normal coronaries and apical ballooning.
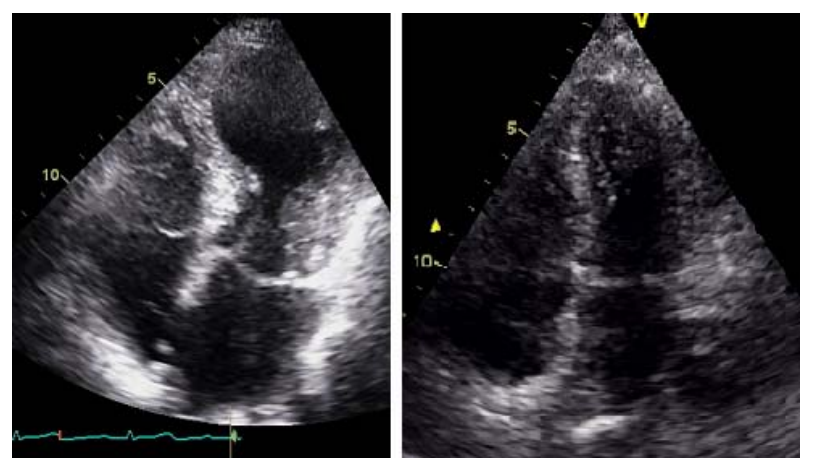

Figure 3. Initial and follow-up echocardiograms showing transient apical ballooning.

in $80 \%$-mild in $60 \%$, moderate in $13 \%$ and severe in $7 \%$.

Clinical course was complicated by heart failure in $37 \%$ of patients, hypotension requiring presser support in 4 patients, atrial fibrillation in 2 patients and ventricular tachycardia and ventricular fibrillation in 1 patient.

Repeat echocardiography (Table 3) was performed at 9 (IQR 5, 23) days (range 4, 26 days). There was a significant improvement in EF from 35\% (IQR 25, 40) on the initial study to $55 \%$ (IQR 52.5, 60) at follow-up (p < 0.0001 , Figure 4). Diastolic dysfunction was present in $27 \%$ - Grade 1 in $20 \%$ and Grade 2 in $7 \%$. Estimated left atrial pressure was 12 (SD 4) $\mathrm{mmHg}$ and estimated pulmonary artery systolic pressure was 30 (SD 10). Mitral regurgitation was present in $47 \%$ - mild in $44 \%$, moder-
Table 3. Initial and follow-up echocardiography.

\begin{tabular}{cccc}
\hline & $\begin{array}{c}\text { Initial } \\
\text { echocardiography }\end{array}$ & $\begin{array}{c}\text { Follow-up } \\
\text { echocardiography }\end{array}$ & p value \\
\hline $\begin{array}{c}\text { Ejection fraction, } \\
\text { median (IQR), } \%\end{array}$ & $35(25,40)$ & $55(52.5,60)$ & $<0.0001$ \\
$\begin{array}{c}\text { Diastolic } \\
\text { dysfunction, \% }\end{array}$ & 72 & 27 & \\
$\begin{array}{c}\text { Estimated left atrial } \\
\text { pressure, mean (SD), } \\
\text { mmHg }\end{array}$ & $14(5)$ & $12(4)$ & \\
$\begin{array}{c}\text { Estimated pulmonary } \\
\text { artery systolic } \\
\text { pressure, mean (SD), } \\
\text { mmHg }\end{array}$ & $38(10)$ & $30(10)$ \\
$\quad \begin{array}{c}\text { Mitral } \\
\text { regurgitation, \% }\end{array}$ & 83 & 47 \\
\hline
\end{tabular}

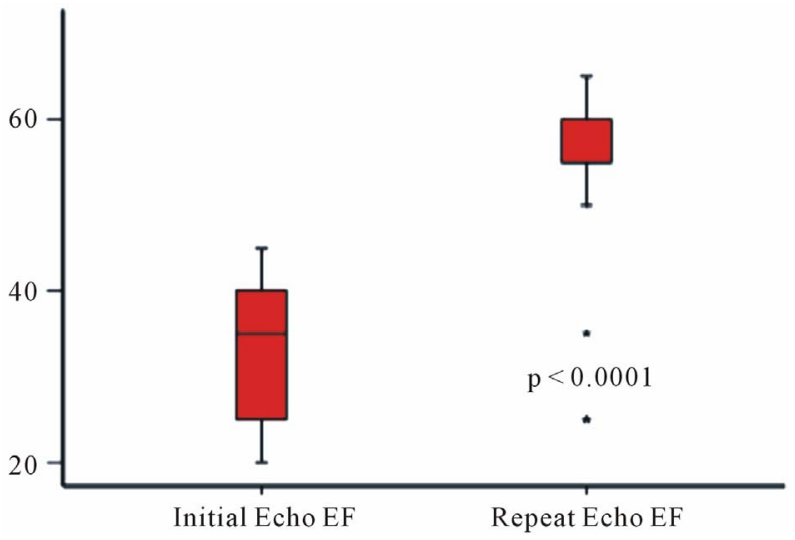

Figure 4. Change from initial to follow-up ejection fraction.

ate in 3\%, while none had severe MR on follow-up.

Correlates of Delta EF (Table 4, Figure 5): Delta EF showed negative correlation with number of leads with $\mathrm{T}$ wave inversion (Spearman's rho $-0.64, p=0.01$ ) and with QT interval (Spearman's rho $-0.51, \mathrm{p}=0.02$ ). Additional analyses were performed to evaluate the robustness of the relationship between the number of leads with 
Table 4. Correlates of delta EF.

\begin{tabular}{ccc}
\hline & Spearman's rho & $\mathrm{p}$ value \\
\hline T wave inversion, mm & -0.25 & 0.4 \\
Number of leads with T wave inversion & -0.65 & 0.01 \\
QT interval & -0.51 & 0.02 \\
Age & -0.03 & 0.9 \\
NT-Pro BNP & 0.29 & 0.4 \\
Peak CPK & 0.29 & 0.3 \\
Peak Troponin I & 0.09 & 0.7 \\
Peak CK-MB & 0.28 & 0.3 \\
Heart rate & 0.30 & 0.2 \\
ST depression, mm & 0.26 & 0.5 \\
Number of leads with ST depression & -0.24 & 0.5 \\
ST elevation, mm & 0.58 & 0.3 \\
Initial EF & 0.22 & 0.7 \\
Number of leads with ST elevation & -0.32 & 0.17 \\
\hline
\end{tabular}

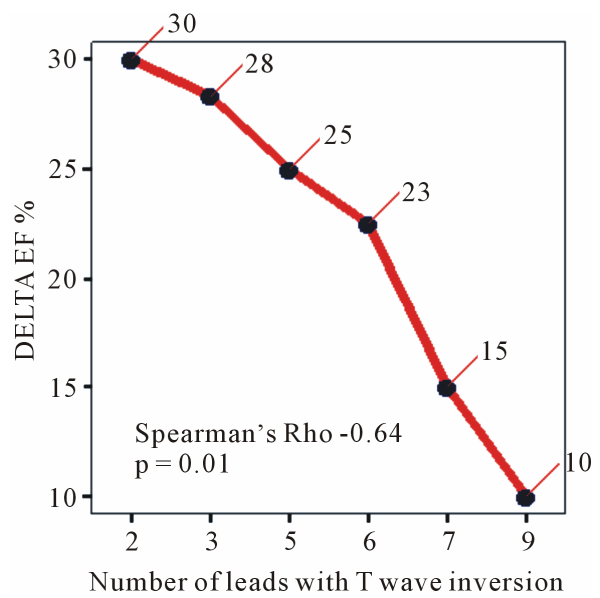

Figure 5. Correlation between delta EF and number of leads with $\mathrm{T}$ wave inversion.

T wave inversion and Delta EF. Importantly, there was no correlation between the number of leads with $T$ wave inversion and the initial EF (Spearman's Rho 0.29, p = $0.25)$. Moreover, there was no correlation between Delta $\mathrm{EF}$ and the duration from initial to follow-up echocardiography (Spearman's Rho $-0.1, \mathrm{p}=0.6$ ). On multivariable analysis (Table 5), the number of leads with $T$ wave inversion was the only variable which was associated with Delta EF (coefficient $-4.878, \mathrm{p}=0.04$ ).

\section{Discussion}

The main finding of this study is that ECG findings at
Table 5. Multivariable correlates of delta EF.

\begin{tabular}{ccc}
\hline & Coefficients & $\mathrm{p}$ value \\
\hline Peak Troponin I & 0.420 & 0.176 \\
Heart rate & 0.020 & 0.811 \\
ST depression, mm & 0.815 & 0.838 \\
Number of leads with ST depression & 1.876 & 0.373 \\
ST elevation, mm & 4.616 & 0.241 \\
Number of leads with ST elevation & -6.462 & 0.147 \\
T wave inversion, mm & 0.797 & 0.605 \\
Number of leads with T wave inversion & -4.892 & 0.036 \\
Initial EF & -0.352 & 0.121 \\
\hline
\end{tabular}

initial presentation correlate with the extent of LV function recovery early after presentation in patients with stress cardiomyopathy.

Stress cardiomyopathy is a reversible cause of LV systolic dysfunction that mimics acute coronary syndrome and is often precipitated by a stressful event. The clinical profile of our series of patients with stress cardiomyopathy is similar to that reported in a systematic review of 14 studies [7]. The mean age of patients in our study was 73.2 years compared to 58 to 77 reported previously [7]. Females accounted for $67 \%$ of patients in our series compared to $88 \%$. A recent study found that precipitating factors in males were more often physical stressors including medical conditions, procedures and treatments, whereas emotional stress was more often noted in females [8]. The greater proportion of physical stressors and the concordant greater proportion of males in our patients likely reflect the demographics of the patient population at our institution. Our institution is a tertiary care multi-specialty hospital located in Miami Beach, which has a higher proportion of persons aged 65 years or older (19.2\%), compared to the national average of $12.9 \%$ (US Census data, accessed at

http://www.census.gov/). Our patient cohort was not only older but also sicker, with a higher representation of physical stressors (medical conditions and interventions). While stress cardiomyopathy has been classically described in female patients with emotional stressors, with increasing awareness of this condition and a higher index of suspicion, it is being increasingly recognized in males with physical stressors $[8,9]$.

Hypertension and diabetes were present in $53 \%$ and $27 \%$ respectively in our series compared to $43 \%$ and $11 \%$. Chest pain and dyspnea were present in $67 \%$ and $20 \%$ respectively in our series compared to $67.8 \%$ and $17.8 \%$. T wave changes were present in $70 \%$ of our patients compared to $64.3 \%$. The pattern of mild cardiac 
biomarker elevation was also similar to that reported previously. The median ejection fraction in our study was $40 \%$ compared to a range of mean ejection fraction of $20 \%$ to $49 \%$ reported previously.

We found a significant improvement in ejection fraction to median of $55 \%$ (IQR 52.5, 60) on follow-up echocardiography at 9 (IQR 5, 23) days. Previous studies have reported improvement in ejection fraction over a period of days to weeks and the mean ejection fraction on follow-up was reported to range between $60 \%$ to $76 \%$ $[7,10]$. In a series of 22 patients, LV function recovery was noted within 5 days in 7 patients and at $24 \pm 29$ days in the overall group [11]. In a series of 6 patients, complete recovery of LV function was seen at $4.5 \pm 1.5$ days [12].

We found that the number of leads with $T$ wave inversion and the QT interval were inversely correlated with the extent of recovery of ejection fraction. On multivariable analysis, only the number of leads with $T$ wave inversion was a significant predictor of improvement in EF. Baseline predictors of LV function recovery have not been specifically addressed by previous studies. The present study does not address potential mechanisms underlying the relationship between ECG changes at presentation and LV function recovery. The pathophysiology of stress cardiomyopathy is not completely understood and has been attributed to catecholamine-induced myocardial dysfunction possibly mediated by metabolic abnormalities and intracellular calcium overload [13-15]. Histological changes consistent with catecholamine-induced myocardial injury have been found in patients with stress cardiomyopathy [14]. Impaired microvascular perfusion has also been implicated in the pathogenesis [15-17]. Despite the finding of elevated cardiac markers and impaired microvascular perfusion, there is no evidence of myocardial necrosis. Recovery of histological changes as well as of perfusion defects and metabolic abnormalities has been demonstrated [14-16].

The pathophysiology of ECG changes and left ventricular function abnormality in stress cardiomyopathy has been explored by several approaches. In one such line of enquiry, ECG changes in stress cardiomyopathy have been contrasted with that of acute ST elevation myocardial infarction $[18,19]$. It has been suggested that the differences reflect subepicardial abnormality in stress cardiomyopathy versus transmural pathology in ST elevation myocardial infarction [20]. Since, ECG changes in ST elevation myocardial infarction reflect myocardial necrosis, (which is not a feature of stress cardiomyopathy), ECG changes classically associated with myocardial stunning [21] are more pertinent to this condition. In particular, T wave inversion and QT interval prolongation have been associated with stunned myocardium and are thought to be mainly caused by prolongation of the action potential [19,22-24].

Electrophysiological studies have suggested that the $\mathrm{T}$-wave abnormalities seen in takotsubo cardiomyopathy are due to abnormal LV repolarization gradients [25]. Experimental studies have suggested that $\mathrm{T}$ wave inversion reflects altered transmural repolarization sequence and is associated with LV diastolic dysfunction [26,27]. $\mathrm{T}$ wave inversion has been proposed to be a marker for reversible left ventricular systolic function [21]. Negative $\mathrm{T}$ wave with QT prolongation has shown to be related to reperfusion injury and stunning of left ventricle [22]. The number of leads with $\mathrm{T}$ wave inversion may possibly reflect the spatial extent of altered reploarization. Whether the spatial extent of altered transmural repolarization sequence is associated with the severity of systolic dysfunction needs to be studied. Further studies may address the relationship between the extent of altered transmural repolarization and LV systolic function recovery in patients with stress cardiomyopathy.

The methodological limitations of the combined retrospective and prospective design are acknowledged. The current findings of the relationship between ECG changes and LV function recovery need to be validated in a larger prospective series. Prospective studies with initial and follow-up echocardiography at pre-specified intervals may be required to better understand the pattern of LV function recovery in patients with stress cardiomyopathy. A limitation in view of the combined retrospective and prospective design of our study is that the duration from symptom onset to initial echocardiography and the interval between initial and repeat echocardiography were not pre-specified. However, we believe that this is not likely to influence the main finding of the study for the following reasons. There was no correlation between the number of leads with $T$ wave inversion and initial EF. Moreover, there was no correlation between Delta EF and the duration from initial to follow-up echocardiography. In addition, multivariable analysis showed an independent association between the number of leads with $T$ wave inversion and Delta EF.

We do not report serial ECG data. The objective of the present study was to evaluate the relationship between ECG findings at initial presentation (typically in the emergency room, within a few hours of symptom onset) and left ventricular function recovery. The initial echocardiogram was obtained at less than 24 hours from presentation. While it would be interesting to study the correlation between serial ECG data with serial changes in left ventricular function, this would also exponentially increase the complexity of the analysis without contributing significantly to the primary objective of risk stratification at initial presentation. 
The findings of this study may potentially inform risk stratification of patients with stress cardiomyopathy and may aid intensive medical management of high risk patients.

\section{Conclusion}

The number of leads with $\mathrm{T}$ inversion is negatively correlated with and is an independent predictor of the extent of improvement of EF in patients with stress cardiomyopathy. Hence the ECG at initial presentation can be used as a simple tool to predict LV function recovery.

\section{REFERENCES}

[1] A. Prasad, A. Lerman and C. S. Rihal, "Apical Ballooning Syndrome (Tako-Tsubo or Stress Cardiomyopathy): A Mimic of Acute Myocardial Infarction," American Heart Journal, Vol. 155, No. 3, 2008, pp. 408-417. doi:10.1016/j.ahj.2007.11.008

[2] G. S. Wagner, P. Macfarlane, H. Wellens, et al., "AHA/ ACCF/HRS Recommendations for the Standardization and Interpretation of the Electrocardiogram: Part VI: Acute Ischemia/Infarction: A Scientific Statement from the American Heart Association Electrocardiography and Arrhythmias Committee, Council on Clinical Cardiology; the American College of Cardiology Foundation; and the Heart Rhythm Society. Endorsed by the International Society for Computerized Electrocardiology," Journal of the American College of Cardiology, Vol. 53, No. 11, 2009, pp. 1003-1011. doi:10.1016/j.jacc.2008.12.016

[3] H. C. Bazett, "An Analysis of the Time-Relations of Electrocardiograms," Annals of Noninvasive Electrocardiology, Vol. 2, No. 2, 1997, pp. 177-194. doi:10.1111/j.1542-474X.1997.tb00325.x

[4] P. M. Rautaharju, B. Surawicz, L. S. Gettes, et al., "AHA/ACCF/HRS Recommendations for the Standardization and Interpretation of the Electrocardiogram: Part IV: The ST Segment, T and U Waves, and the QT Interval: A Scientific Statement from the American Heart Association Electrocardiography and Arrhythmias Committee, Council on Clinical Cardiology; the American College of Cardiology Foundation; and the Heart Rhythm Society: Endorsed by the International Society for Computerized Electrocardiology," Circulation, Vol. 119, No. 10, 2009, pp. e241-e250. doi:10.1161/CIRCULATIONAHA.108.191096

[5] R. M. Lang, M. Bierig, R. B. Devereux, et al., "Recommendations for Chamber Quantification: A Report from the American Society of Echocardiography's Guidelines and Standards Committee and the Chamber Quantification Writing Group, Developed in Conjunction with the European Association of Echocardiography, a Branch of the European Society of Cardiology," Journal of the American Society of Echocardiography, Vol. 18, No. 12, 2005, pp. 1440-1463. doi:10.1016/j.echo.2005.10.005

[6] M. A. Quinones, C. M. Otto, M. Stoddard, A. Waggoner and W. A. Zoghbi, "Recommendations for Quantification of Doppler Echocardiography: A Report from the Doppler Quantification Task Force of the Nomenclature and Standards Committee of the American Society of Echocardiography," Journal of the American Society of Echocardiography, Vol. 15, No. 2, 2002, pp. 167-184. doi:10.1067/mje.2002.120202

[7] M. Gianni, F. Dentali, A. M. Grandi, G. Sumner, R. Hiralal and E. Lonn, "Apical Ballooning Syndrome or Takotsubo Cardiomyopathy: A Systematic Review," European Heart Journal, Vol. 27, No. 13, 2006, pp. 15231529. doi:10.1093/eurheartj/ehl032

[8] S. Kurisu, I. Inoue, T. Kawagoe, et al., "Presentation of Tako-Tsubo Cardiomyopathy in Men and Women," Clinical Cardiology, Vol. 33, No. 1, 2010, pp. 42-45. doi:10.1002/clc. 20700

[9] S. W. Sharkey, D. C. Windenburg, J. R. Lesser, et al., "Natural History and Expansive Clinical Profile of Stress (Tako-Tsubo) Cardiomyopathy," Journal of the American College of Cardiology, Vol. 55, No. 4, 2010, pp. 333-341. doi:10.1016/j.jacc.2009.08.057

[10] K. Tsuchihashi, K. Ueshima, T. Uchida, et al., "Transient Left Ventricular Apical Ballooning without Coronary Artery Stenosis: A Novel Heart Syndrome Mimicking Acute Myocardial Infarction. Angina Pectoris-Myocardial Infarction Investigations in Japan," Journal of the American College of Cardiology, Vol. 38, No. 1, 2001, pp. 11-18. doi:10.1016/S0735-1097(01)01316-X

[11] S. W. Sharkey, J. R. Lesser, A. G. Zenovich, et al., "Acute and Reversible Cardiomyopathy Provoked by Stress in Women from the United States," Circulation, Vol. 111, 2005, pp. 472-479. doi:10.1161/01.CIR.0000153801.51470.EB

[12] F. Cangella, A. Medolla, G. De Fazio, et al., "Stress Induced Cardiomyopathy Presenting as Acute Coronary Syndrome: Tako-Tsubo in Mercogliano, Southern Italy," Cardiovasc Ultrasound, Vol. 5, 2007, p. 36. doi:10.1186/1476-7120-5-36

[13] I. S. Wittstein, D. R. Thiemann, J. A. Lima, et al., "Neurohumoral Features of Myocardial Stunning Due to Sudden Emotional Stress," The New England Journal of Medicine, Vol. 352, 2005, pp. 539-548. doi:10.1056/NEJMoa043046

[14] H. M. Nef, H. Mollmann, S. Kostin, et al., "Tako-Tsubo Cardiomyopathy: Intraindividual Structural Analysis in the Acute Phase and after Functional Recovery," European Heart Journal, Vol. 28, No. 20, 2007, pp. $2456-$ 2464. doi:10.1093/eurheartj/eh1570

[15] S. Kurisu, I. Inoue, T. Kawagoe, et al., "Myocardial Perfusion and Fatty Acid Metabolism in Patients with Tako-Tsubo-Like Left Ventricular Dysfunction," Journal of the American College of Cardiology, Vol. 41, No. 5, 2003, pp. 743-748. doi:10.1016/S0735-1097(02)02924-8

[16] K. Ito, H. Sugihara, T. Kawasaki, et al., "Assessment of Ampulla (Takotsubo) Cardiomyopathy with Coronary Angiography, Two-Dimensional Echocardiography and 99mtc-Tetrofosmin Myocardial Single Photon Emission Computed Tomography," Annals of Nuclear Medicine, Vol. 15, No. 4, 2001, pp. 351-355. 


\section{doi:10.1007/BF02988242}

[17] J. Ako, K. Takenaka, K. Uno, et al., "Reversible Left Ventricular Systolic Dysfunction-Reversibility of Coronary Microvascular Abnormality," Japanese Heart Journal, Vol. 42, No. 3, 2001, pp. 355-363. doi:10.1536/jhj.42.355

[18] K. A. Bybee, A. Motiei, I. S. Syed, et al., "Electrocardiography Cannot Reliably Differentiate Transient Left Ventricular Apical Ballooning Syndrome from Anterior ST-Segment Elevation Myocardial Infarction," Journal of Electrocardiology, Vol. 40, No. 1, 2007, pp. e1-e6.

[19] S. W. Sharkey, "Electrocardiogram Mimics of Acute ST-Segment Elevation Myocardial Infarction: Insights from Cardiac Magnetic Resonance Imaging in Patients with Tako-Tsubo (Stress) Cardiomyopathy," Journal of Electrocardiology, Vol. 41, No. 6, 2008, pp. 621-625. doi:10.1016/j.jelectrocard.2008.06.015

[20] S. Sclarovsky and K. Nikus, "The Electrocardiographic Paradox of Tako-Tsubo Cardiomyopathy-Comparison with Acute Ischemic Syndromes and Consideration of Molecular Biology and Electrophysiology to Understand the Electrical-Mechanical Mismatching," Journal of Electrocardiology, Vol. 43, No. 2, 2010, pp. 173-176. doi:10.1016/j.jelectrocard.2009.07.015

[21] R. A. Kloner, "Inverted T Waves. An Electrocardiographic Marker of Stunned or Hibernating Myocardium in Man?" Circulation, Vol. 82, No. 3, 1990, pp. 1060-1061. doi:10.1161/01.CIR.82.3.1060

[22] Y. Hirota, Y. Kita, R. Tsuji, et al., "Prominent Negative T Waves with QT Prolongation Indicate Reperfusion Injury and Myocardial Stunning," Journal of Cardiology, Vol. 22, No. 2-3, 1992, pp. 325-340.

[23] S. Kurisu, I. Inoue, T. Kawagoe, et al., "Time Course of Electrocardiographic Changes in Patients with TakoTsubo Syndrome: Comparison with Acute Myocardial Infarction with Minimal Enzymatic Release," Circulation Journal, Vol. 68, No. 1, 2004, pp. 77-81. doi:10.1253/circj.68.77

[24] R. Ogura, Y. Hiasa, T. Takahashi, et al., "Specific Findings of the Standard 12-Lead ECG in Patients with 'Takotsubo' Cardiomyopathy: Comparison with the Findings of Acute Anterior Myocardial Infarction," Circulation Journal, Vol. 67, No. 8, 2003, pp. 687-690. doi:10.1253/circj.67.687

[25] H. Furushima, M. Chinushi, A. Sanada and Y. Aizawa, "Ventricular Repolarization Gradients in a Patient with Takotsubo Cardiomyopathy," Europace, Vol. 10, No. 9, 2008, pp. 1112-1115. doi:10.1093/europace/eun166

[26] T. G. Zhu, C. Patel, S. Martin, et al., "Ventricular Transmural Repolarization Sequence: Its Relationship with Ventricular Relaxation and Role in Ventricular Diastolic Function," European Heart Journal, Vol. 30, No. 3, 2009, pp. 372-380. doi:10.1093/eurheartj/ehn585

[27] P. Ofman, J. R. Cook, L. Navaravong, et al., "T-Wave Inversion and Diastolic Dysfunction in Patients with Electrocardiographic Left Ventricular Hypertrophy," Journal of Electrocardiology, Vol. 45, No. 6, 2012, pp. 764-769. doi:10.1016/j.jelectrocard.2012.06.001 